\title{
[gw22-e0662] CHRONIC ISCHEMIA SUPPRESSED THE EXPRESSION OF KCNO1 PROTEIN AND COMPROMISED THE REPOLARISATION RESERVE
}

Guo Xiaogang, Gao Xiuren, Tang Lilong, Peng Longyun, He Xuyu, Zhu YingyingFirst Affiliated Hospital, Sun Yat-sen University, Guangzhou, China

10.1136/heartjnl-2011-300867.10

Objectives To explore the impact of chronic or transient ischemia on slowly activated delayed rectifier potassium current (IKs) channel subunit expression and its subsequent effect.

Methods $\mathrm{I}_{\mathrm{K} s}$ channel subunit expression was examined following transient ischemia or permanent infarction and changes in monophasic action potential (MAP) waveforms challenged by $\beta$-adrenergic stimulation were evaluated using a rabbit model of transient or chronic cardiac ischemia for two or five days.

Results The epicardial MAP of peri-infarct zone of animal subjected to infarction for two days showed a less shortening of MAPD90 (MAPD90 shortened by $10.6 \pm 7.2$ vs $28.9 \pm 8.3$ of control groups, $\mathrm{p}<0.05)$ and an increased triangulation (78.1 \pm 11.3 vs $45.5 \pm 6.9$ of control group, $p<0.05$ ) under adrenergic stimulation. Concomitantly, the median number of premature ventricular beats of this group was 36 (vs 9 of control group, $\mathrm{p}<0.05)$. KCNQ1 protein of peri-infarct tissue harvested from peri-infarct zone by day 2 was down-regulated by approximately $80 \%$ compared with that of control group $(\mathrm{p}<0.05)$.

Conclusion Chronic ischemia increased the incidence of ventricular arrhythmias under adrenergic stimulation and was associated with increased MAP triangulation of the periinfarct zone. Downregulation of KCNO1 protein may be the underlying cause of these changes. 